\title{
Analysis of Salmonella enterica serovar Enteritidis isolates from chickens and chicken meat products in Malaysia using PFGE, and MLST
}

Zunita Zakaria ${ }^{1,2^{*}}$ D, Latiffah Hassan ${ }^{2,3}$, Zawiyah Sharif ${ }^{4}$, Norazah Ahmad $^{5}$, Rohaya Mohd Ali ${ }^{6}$, Suraya Amir Husin ${ }^{5}$, Nor Hazrin binti Abd Hazis ${ }^{5}$, Nor Fitriah Mohamed Sohaimi ${ }^{2}$, Shafini Abu Bakar ${ }^{4}$ and Bashiru Garba ${ }^{1,7}$

\begin{abstract}
Background: Salmonella is a very important foodborne pathogen causing illness in humans. The emergence of drug-resistant strains also constitutes a serious worry to global health and livestock productivity. This study investigated Salmonella isolates from chicken and chicken meat products using the phenotypic antimicrobial screening as well as the molecular characteristics of Salmonella isolates. Upon serotyping of the isolates, the antimicrobial susceptibility profiling using a panel of 9 commonly used antimicrobials was done. Subsequently, the molecular profiles of all the isolates were further determined using Pulsed Field Gel Electrophoresis (PFGE) and the Whole Genome Multi-Locus Sequence Type (wgMLST) analysis in order to obtain the sequence types.

Results: The PFGE data was input into FPQuest software, and the dendrogram generated was studied for possible genetic relatedness among the isolates. All the isolates were found to belong to the Salmonella Enteritidis serotype with notable resistance to tetracycline, gentamycin, streptomycin, and sulfadimidine. The $S$. Enteritidis isolates tested predominantly subtyped into the ST11 and ST1925, which was found to be a single cell variant of ST11. The STs were found to occur in chicken meats, foods, and live chicken cloacal swabs, which may indicate the persistence of the bacteria in multiple foci.
\end{abstract}

Conclusion: The data demonstrate the presence of S. Enteritidis among chickens, indicating its preference and reservoir status for enteric Salmonella pathogens.

Keywords: Salmonella Enteritidis, Chicken meat products, Pulse field gel electrophoresis, Multi-locus sequence typing, Whole-genome sequencing, Antimicrobial resistance

\footnotetext{
* Correspondence: zunita@upm.edu.my

The work was carried out at the Bacteriology Laboratory

'Institute of Bioscience, Universiti Putra Malaysia, 43400 Serdang, Selangor,

Malaysia

${ }^{2}$ Bacteriology Laboratory, Department of Veterinary Pathology and

Microbiology, Faculty of Veterinary Medicine, Universiti Putra Malaysia, 43400

Serdang, Selangor, Malaysia

Full list of author information is available at the end of the article
}

(C) The Author(s). 2020 Open Access This article is licensed under a Creative Commons Attribution 4.0 International License, which permits use, sharing, adaptation, distribution and reproduction in any medium or format, as long as you give appropriate credit to the original author(s) and the source, provide a link to the Creative Commons licence, and indicate if changes were made. The images or other third party material in this article are included in the article's Creative Commons licence, unless indicated otherwise in a credit line to the material. If material is not included in the article's Creative Commons licence and your intended use is not permitted by statutory regulation or exceeds the permitted use, you will need to obtain permission directly from the copyright holder. To view a copy of this licence, visit http://creativecommons.org/licenses/by/4.0/ The Creative Commons Public Domain Dedication waiver (http://creativecommons.org/publicdomain/zero/1.0/) applies to the data made available in this article, unless otherwise stated in a credit line to the data. 


\section{Background}

The continuous emergence of multidrug-resistant strains of non-typhoidal Salmonella constitutes a serious health hazard globally [1-4]. In recent years, Salmonella enterica associated with gastrointestinal infection in humans has been reported with increasing frequency worldwide [5]. $S$. Enteritidis is one of the most common causes of foodborne infection in humans [6]. While the majority of the infections are mild self-limiting illnesses, a small number have been reported to cause invasive infections, which is characterized by severe illness that requires hospitalization [7]. The popularity of $S$. Enteritidis is attributed to the unique ability of this serotype to contaminate chicken egg and meat without any discernible illness to the chickens [8]. Furthermore, multiple investigations have identified antimicrobial resistance phenotypes of $S$. Enteritidis from among various food materials of poultry origin [9].

In Malaysia, retail chicken meat has been reported as a popular source of multiple antimicrobial-resistant Salmonella with $S$. Enteritidis accounting for 6.7\% [10]. This multidrug-resistant (MDR) Salmonella are considered a serious public health problem due to tendencies for transmission of resistance to humans across the poultry production chain; thus it has become paramount to identify and characterize this important pathogen [11]. Moreover, concerns over the emergence of Salmonella with increased virulence, transmissibility, and antibioticresistance features, has necessitated the need for highly efficient methods that can identify these variant pathogens to track their spread especially across the human, animal and environmental interface [12]. In this regard, molecular techniques including whole genome sequencing, Pulse Field Gel Electrophoresis (PFGE), and Multi-Locus Sequence Typing (MLST) are among the commonly employed methods. These techniques can characterize pathogens in order to determine clonal and strain distribution across various environments and hosts.

Pulsed-field gel electrophoresis is one of the most widely used methods for the epidemiological studies of pathogenic bacterial organisms due to its high discriminatory ability [13]. With the globalization of trade, including poultry and poultry products, PFGE can be useful in understanding the diversity and evolution of infectious disease agents in order to evaluate their genetic relatedness to determine their point source during epidemiological investigations [14]. The principle of this method is based on the restriction enzyme digestion of whole DNA to produce fragment patterns that vary from strain to strain. The method relies on the distinct genomic differences between isolates that are observed as a result of the rapid accumulation of genetic variations that lead to slightly detectable differences between DNA fingerprints patterns within a clone [12].
Multi-Locus Sequence Typing (MLST) analysis, on the other hand, is best suited for longer-term as well as global epidemiologic investigations of infectious disease agents [12]. It is based on the principle of the multiLocus enzyme electrophoresis that uses a combination of discriminatory power and clonal stability, which has proven to be extremely efficient in characterizing clones within a population of bacterial organisms causing serious disease [15]. However, it uses allelic differences in the sequence of various house-keeping genes that are often exploited to differentiate strains [16].

In this investigation, the phenotypic antimicrobial resistance profile and molecular characteristics of $S$. Enteritidis isolated from chicken and food isolates was assessed using Pulsed-field gel electrophoresis and Multi-Locus Sequence Typing (MLST) analysis in order to understand the molecular characteristics and the epidemiological distribution of the $S$. Enteritidis strains circulating in the Central region of Peninsular Malaysia from 2016 to 2018. This will help reveal the genetic relatedness and strain variabilities and distribution among the antimicrobial resistant isolates obtained from human clinical samples, live birds, and chicken meat in Malaysia.

\section{Results}

Serotyping of Salmonella and antimicrobial resistance

All of the 47 isolates were analyzed by the classical serotyping slide agglutination test comprising of ready-to-eat foods (7), chicken meats (11) and chicken cloacal swabs (29), in accordance with the White-Kauffmann-Le Minor scheme and only isolates belonging to the $S$. Enteritidis serotype were selected for this study. Based on the phenotypic antimicrobial resistance pattern of the isolates against the nine (9) different antimicrobial drugs, 27 (57.45\%) of the isolates were found to show resistance to 1 or more antimicrobials tested (Table 1). However, out of the 20 that were susceptible to the drugs tested, 6 showed intermediate resistance to ampicillin, streptomycin, and tetracycline.

When stratified by the source of samples, $S$. Enteritidis isolated from food samples exhibited the highest number of resistance 5/7 (71.4\%), followed by cloacal swabs 17/29 (58.6\%) and then chicken meats with 5/11 (45.5\%). However, chicken swab isolates had the overall highest resistance with $17 / 47$ (36.2\%). Importantly, only two isolates, both from cloacal swab samples, showed multi-drug resistance $(\mathrm{CCS} 08=\mathrm{AMP}$; $\mathrm{CN}$; TE; CCS025 = TE, S, AMP). Moreover, the antimicrobial agent with the most resistance across all the isolates tested was tetracycline 21/47 (46.8\%); While ampicillin had $7 / 47$ (14.8\%) and, streptomycin, sulfadimidine/trimethoprim, and gentamycin all had 1/47 (2.1\%) respectively.

\section{Multi-locus sequence typing analysis}

For the MLST of the completely sequenced bacterial genomes, short sequence reads were first assembled to 
Table 1 Antimicrobial resistance pattern of the 47 Salmonella Enteritidis isolates

\begin{tabular}{|c|c|c|c|c|}
\hline New ID & Source & AMR & ST & Remark \\
\hline$\overline{C M \& M P 01}$ & Chicken meat & - & 1925 & Susceptible \\
\hline CM\&MP02 & Chicken meat & TE & 1925 & Tetracycline resistant \\
\hline CM\&MP03 & Food & - & 11 & Susceptible \\
\hline CM\&MP04 & Food & $\mathrm{TE}$ & 1925 & Tetracycline resistant \\
\hline CM\&MP05 & Food & - & 11 & Susceptible \\
\hline CM\&MP06 & Food & TE & 1925 & Tetracycline resistant \\
\hline CM\&MP07 & Food & TE & 1925 & Tetracycline resistant \\
\hline CM\&MP08 & Chicken meat & - & 11 & Susceptible \\
\hline CM\&MP09 & Chicken meat & TE & 1925 & Tetracycline resistant \\
\hline CM\&MP010 & Chicken meat & $\mathrm{TE}$ & 1925 & Tetracycline resistant \\
\hline CM\&MP011 & Food & TE & 1925 & Tetracycline resistant \\
\hline CM\&MP012 & Food & TE & 1925 & Tetracycline resistant \\
\hline CM\&MP013 & Chicken meat & - & 1925 & Susceptible \\
\hline CM\&MP014 & Chicken meat & TE & 1925 & Tetracycline resistant \\
\hline CM\&MP015 & Chicken meat & - & 11 & Susceptible \\
\hline CM\&MP016 & Chicken meat & - & 1925 & Susceptible \\
\hline CM\&MP017 & Chicken meat & - & 1925 & Susceptible \\
\hline CM\&MP018 & Chicken meat & SXT & 292 & Sulfamethazine/Trimeth \\
\hline CCSO1 & Chicken swab & TE & 1925 & Tetracycline resistant \\
\hline $\mathrm{CCSO} 2$ & Chicken swab & TE & 1925 & Tetracycline resistant \\
\hline CCSO3 & Chicken swab & - & 1925 & Susceptible \\
\hline CCSO4 & Chicken swab & - & 11 & Susceptible \\
\hline CCSO5 & Chicken swab & TE & 11 & Tetracycline resistant \\
\hline CCSO6 & Chicken swab & - & 11 & Susceptible \\
\hline CCSO7 & Chicken swab & AMP & 11 & Ampicillin resistant \\
\hline CCSO8 & Chicken swab & AMP;CN;TE & 11 & Multidrug resistant \\
\hline CCSO9 & Chicken swab & TE & 1925 & Tetracycline resistant \\
\hline CCS010 & Chicken swab & AMP & 11 & Ampicillin resistant \\
\hline CCS011 & Chicken swab & $\mathrm{TE}$ & 1925 & Tetracycline resistant \\
\hline CCS012 & Chicken swab & AMP & 11 & Ampicillin resistant \\
\hline CCSO13 & Chicken swab & - & 1925 & Susceptible \\
\hline CCSO14 & Chicken swab & - & 1925 & Susceptible \\
\hline CCS015 & Chicken swab & - & 1925 & Susceptible \\
\hline CCS016 & Chicken swab & - & 329 & Susceptible \\
\hline CCS017 & Chicken swab & - & 1925 & Susceptible \\
\hline CCS018 & Chicken swab & $\mathrm{TE}$ & 1925 & Tetracycline resistant \\
\hline CCS019 & Chicken swab & AMP & 11 & Ampicillin resistant \\
\hline CCSO20 & Chicken swab & AMP & 11 & Ampicillin resistant \\
\hline CCSO21 & Chicken swab & - & 365 & Susceptible \\
\hline CCS022 & Chicken swab & - & 1925 & Susceptible \\
\hline CCSO23 & Chicken swab & - & 1925 & Susceptible \\
\hline CCSO24 & Chicken swab & - & 1925 & Susceptible \\
\hline
\end{tabular}


Table 1 Antimicrobial resistance pattern of the 47 Salmonella Enteritidis isolates (Continued)

\begin{tabular}{lllll}
\hline New ID & Source & AMR & ST & Remark \\
\hline CCS027 & Chicken swab & TE & 1925 & Tetracycline resistant \\
CCS028 & Chicken swab & TE & 1925 & Tetracycline resistant \\
CCS029 & Chicken swab & TE & 1925 & Tetracycline resistant \\
\hline
\end{tabular}

Key: CM\&MP-Chicken Meat \& Meat Products; CCS-Chicken Cloacal Swab

draft genomes [17]. For the Whole Genome MLST scheme, the MLST allele of each locus was aligned to the genome using BLAST. After that, the ST was determined by a combination of the MLST alleles after closematching of the selected alleles. The MLST typing of all the 47 isolates was based on the comparison of internal sequences of the Salmonella seven housekeeping gene fragments (aroC, dnaN, hemD, hisD, purE, sucA, and thrA). The $47 \mathrm{~S}$. Enteritidis were subtyped into six (6) different STs, with the majority assigned to ST1925 (30) followed by ST11 (12), with ST292, ST365, ST329 and ST2132 assigned to only one isolate each. Worthy of note is the fact that ST1925 is a single locus variant of ST11. Additionally, while ST1925 and ST11 occurred in food materials, chicken meats, and cloacal swabs, ST292 was found only in the chicken while ST365, ST329 and ST2132 were all found in cloacal swabs.

\section{Pulse-field gel electrophoresis}

The XbaI digestion was successfully performed on all the isolates except CCS016 and CCS025 (both from cloacal swabs), which were not typeable by PFGE, hence were excluded. However, the remaining 45 selected isolated yielded 9-13 DNA bands. With a Dice Coefficient of 0.5 and a similarity index of $90 \%$, the PFGE analysis produced ten pulsotypes (1-10) with pulsotypes 6 and 8 being the major ones, pulsotype 1 and 5 had 3 and 2 isolates from chicken and meat isolates while $2,3,4,9$, and 10 appeared as singletons with $100 \%$ similarity. Moreover, the majority of the strains $(17 ; 37.7 \%)$ belonged to pulsotype 6 and 8 . Within the pulsotype 6 , isolates from chicken meats, foods and cloacal swabs exhibited genetic relatedness ranging from 88.9 to $100 \%$, likewise isolates in the pulsotype 8 shared a similarity score in the region of $92-100 \%$ (Fig. 1).

\section{Discriminatory ability}

Simpson's index of diversity $(D)$ was used to compare the bacterial typing method based on MLST and PFGE pattern of the isolates. For the 10 PFGE types, $D$ was 0.96 while for the six (6) sequence types identified by MLST, $D$ was 0.99 . These indices imply that if two isolates are to be sampled randomly from the population, then 96 and $99 \%$ of the time they will be assigned into different types. However, it is recommended that a good index should be greater than $0.95[18,19]$.

\section{Discussion}

This investigation was undertaken to examine Salmonella isolates from foods sold at restaurants, chicken meats sold at supermarkets and wet night market in the central region of Peninsular Malaysia, as well as samples from live chickens from selected poultry farms located within the central region of Peninsular Malaysia in order to assess the antimicrobial susceptibility and the genetic relatedness of the Salmonella pathogen. In total, $47 \mathrm{~S}$. Enteritidis were identified after the culture, isolation, biochemical characterization, and serotyping was done. In order to determine their genetic relatedness, whole-genome sequencing wgMLST and PFGE were conducted.

The antimicrobial susceptibility analysis of all isolates from the food sources, chicken meats, and chicken cloacal swabs exhibited susceptibility and varying resistance characteristics to the antimicrobial panel tested. As mentioned above, all of the 47 isolates were confirmed to be $S$. Enteritidis serotype upon slide agglutination test. The phenotypic antimicrobial resistance result showed that the majority of the isolates were resistant $(57.45 \%)$ to the antimicrobials tested. Although only two isolates had multiple resistance (resistant to 3 or more), the majority of the isolates were resistant to tetracycline. The two Salmonella isolates with a multi-drug resistant profile were resistant to tetracycline, which has gained popularity as a clinically and agriculturally relevant antibiotic [20]. The National Pharmaceutical Regulatory Agency (NPRA), which is the drug control authority of Malaysia under the Ministry of Health (MOH), Malaysia and the Department of Veterinary Services (DVS) under the Ministry of Agriculture has granted approvals for the use of tetracycline in the treatment of disease, as prophylaxis and as growth promoters [21]. However, the inappropriate use of these antibiotics in the foodproducing animals constitutes a serious public health hazard [22]. Moreover, the emergence of multiple antibiotic resistance, as observed in two isolates in this study, may progressively undermine the viability of many of the routinely used antibiotics.

Sequence analysis of the isolates found that the most common sequence type (ST) observed among all the isolates were ST1925 (30) followed by ST11 (12). While novel STs reported in Malaysia for the first time (ST292, ST365, ST329, and ST2132) were also detected in only one isolate for each. Comparison of our result against 


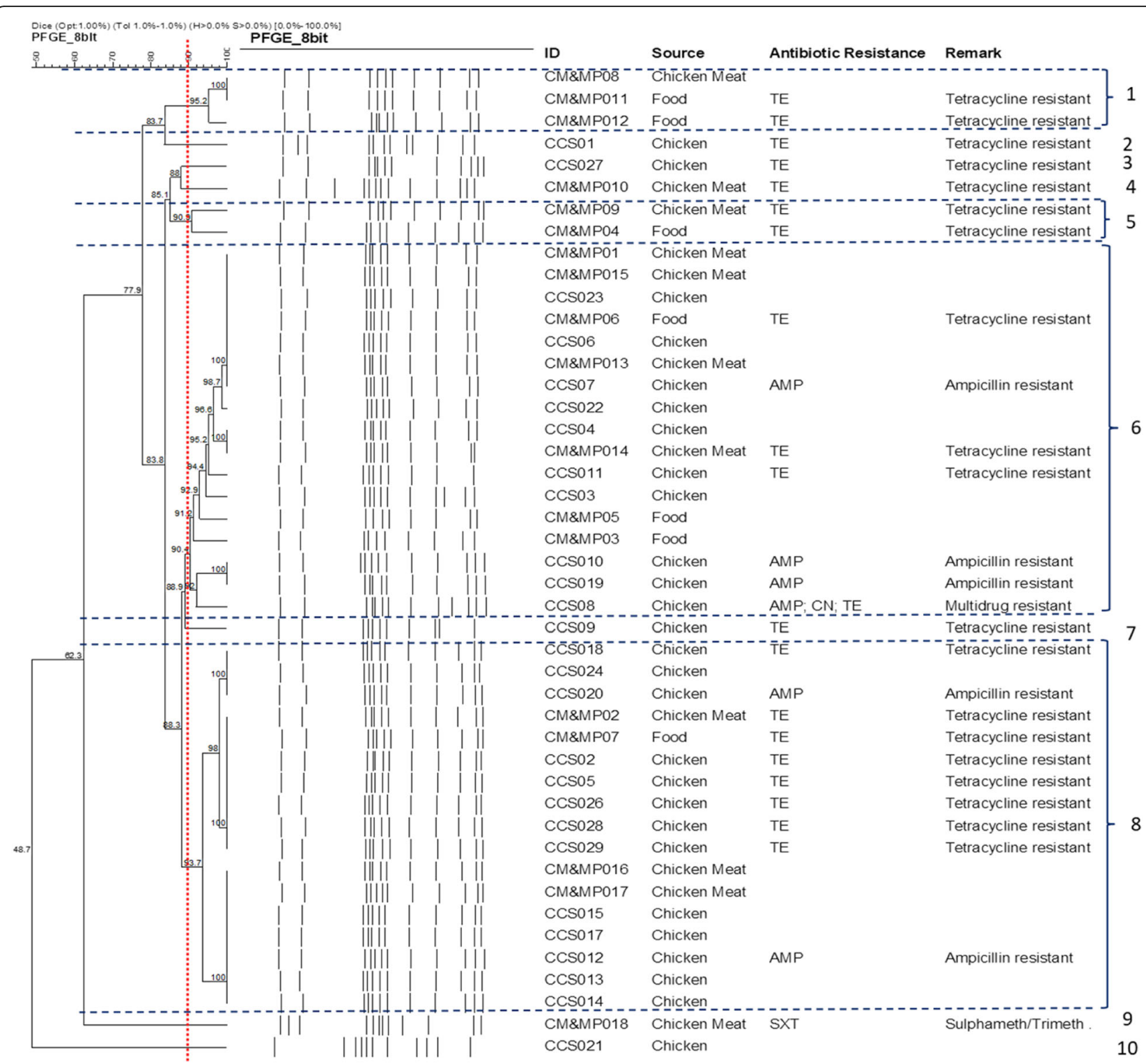

Fig. 1 Pulsed-field gel electrophoresis (PFGE) patterns showing the DNA of the chicken meats, foods and chicken cloacal swab isolates digested by Xbal restriction enzyme $(n=45)$. Nine antimicrobial susceptibility patterns and 10 PFGE pulsotypes $(1-10)$ were identified among the 45 isolates

the MLST database indicates that ST1925 is relatively common among $S$. Enteritidis isolated from human and avian species from the United Kingdom, United States, Australia, and Malaysia (enterobase). While ST11 had been reported in humans, poultry, food, and some wild animal species, including reptiles in many countries from Asia, Africa, South America, and European countries (enterobase). Notably, ST1925 has been reported to be a single cell variant of ST11, and both sequence types are known to be geographically widespread and have previously been reported in foods, humans and animals $[23,24]$. The detection of these STs from different sources may also indicate their ability to adapt to, and persist in different hosts or types of samples. The detection of ST11 in foods may imply possible transmission from neighboring countries like Singapore and China, where the ST type prevails [24]. In this study, the Whole-Genome Sequencing platform was used for the MLST analysis against the traditional PCR based MLST, followed by Sanger sequencing. This is because of its superior discriminatory power and efficiency in genetic detection of variability between isolates, in addition to the fact that the traditional method is both costly and timeconsuming [17]. Furthermore, ST292, ST365, and ST2132 have been previously reported in Singapore (aquatic), India (human \& environmental samples), and 
United States (poultry) while ST329 was reported among isolates obtained from poultry feed in Peru [25-28]. In this study, ST292 was detected in chicken meat, while ST365, ST329 and ST2132 were detected in the chicken cloacal swabs. When analyzed in the MLST database, these strains were not as common as the other STs detected except for ST365, and ST2135 which were found in poultry in the US from Salmonella isolates belonging to the Kentucky serotype. In addition, ST292 and ST2132 showed resistance to sulfadimidine/trimethoprim as well as streptomycin and tetracycline respectively. Therefore, having demonstrated similar antimicrobial susceptibility profiles as well as a common source, this may be suggestive of possible strain relatedness, and potential for transmission between chicken and ready-to-eat foods.

The pulsed-field gel (PFGE) analysis of the isolates revealed that most of the $S$. Enteritidis isolates examined exhibited unique genetic relatedness, albeit with some variability. Cluster analysis identified ten (10) pulsotypes with the majority belonging to pulsotype 6 and 8 , which further had 17 subtypes that shared 100\% identity pattern between the poultry and food source. From the results of the PFGE analysis, it was evident that PFGE revealed more significant differentiation (10 profiles) compared to the MLST, which produced six (6) sequence types. The earlier observation supports this finding that the diversity indices with PFGE produced the highest rate of variability over MLST and the phenotypic antimicrobial susceptibility testing [29]. On the other hand, a one-to-one correlation between PFGE types and ST revealed that some isolates belonging to the same PFGE type had multiple STs and vice versa. The result of this study showed that both MLST and PFGE had a high index of discrimination $(D=0.95)$ above the recommended value. The slight disparity with respect to the 'Simpson's index between MLST and PFGE observed in this study has previously been reported where MLST was found to exhibit higher discriminatory power with respect to the typing of Extended Spectrum Beta Lactamase Escherichia coli [19]. The authors argued that such disparity might be attributed to the spectrum of changes detected by PFGE and MLST. In other words, while PFGE detects changes in nucleotide sequence associated with insertions or deletions of DNA, MLST typing detects nucleotide changes within an amplified gene fragment [19]. However, in recent years, the potentials of molecular techniques in discriminating between strains of $S$. Enteritidis have become more pronounced. Methods with the highest discriminatory power are more specific and therefore, better recommended during investigations of closely related isolates [30]. Furthermore, the PFGE analysis delineated the genetic variability between the $S$. Enteritidis isolates from a different source based on the distinct DNA fingerprints generated. Except for two isolates, all other isolates were type-able and the technique reproducible, which could be very useful as an epidemiological tool for disease outbreak investigation. The present study also showed that various PFGE subtypes identified are present in both fresh chicken meat, live birds, and even cooked food ready for eating. Although the DNA profiles of most of the $S$. Enteritidis isolates from various sources differed, which may indicate that the isolates belong to different clones as revealed by the MLST analysis.

\section{Conclusion}

This investigation has highlighted the usefulness of molecular and phenotype analysis in understanding the genotypic and phenotypic characteristics of $S$. Enteritidis. A higher level of diversity was observed among the $S$. Enteritidis isolates based on the PFGE, which is an indication of its potential as the molecular choice technique for the subtyping of isolates of the same serovar. The five sequence types detected in the present study with the wgMLST analysis also showed host variability by occurring in live chickens, cooked foods, and fresh chicken carcasses in addition to the other animal and human hosts upon comparison with the MLST database. The antimicrobial resistance pattern was equally evident in food isolates as compared to the other sources investigated in this study.

\section{Methods}

\section{Salmonella isolates}

All the Salmonella isolates used in this study were obtained from the laboratory collection of the Food Safety Division, Ministry of Health Malaysia (7), and the Department of Veterinary Services Malaysia (40). After the differential culture, isolation and biochemical characterization, all the isolates were suspended in a Brain Heart Infusion (Oxoid) broth supplemented with $20 \%$ glycerol and then stored at $-80^{\circ} \mathrm{C}$ until required. All the isolates (47) comprised of 29 cloacal swab, seven ready -to-eat foods and 11 fresh chicken portions of meat at retail outlets.

\section{Serotyping and antimicrobial susceptibility testing}

The Salmonella serotype (S. Enteritidis) was determined using the slide agglutination assay according to the Kauffman-White scheme based on the agglutination of the bacteria with commercial Salmonella O (somatic) and $\mathrm{H}$ (flagellar) antisera (DIFCO, Detroit, Mich., USA) in order to identify variants of the $\mathrm{O}$ and $\mathrm{H}$ antigens. While the antimicrobial susceptibility tests were conducted using the disk diffusion method per the Clinical Laboratory Standard Institute (CLSI) protocol [31]. The isolates were tested against ampicillin (Amp), chloramphenicol $(\mathrm{C})$, gentamicin $(\mathrm{CN})$, streptomycin $(\mathrm{S})$, 
sulfamethazine/trimethoprim (SXT), tetracycline (TE), ceftiofur (EFT), cefotaxime (CTX), and ciprofloxacin (CIP).

\section{Whole-genome sequencing and MLST}

The QIAamp DNA Mini Kit (Qiagen, Hilden, Germany) was used to extract and purify the genomic DNA from all the $S$. Enteritidis isolates. While the NGS library preparation was achieved with the aid of the Nextera XT DNA Sequencing Library Preparation Kit (FC-131-1096; Illumina, San Diego, California, USA) following the manufacturer's instructions, and the sequencing was done using the Illumina NextSeq sequencer by scanning for adapter sequences followed by removal of low-quality sequences. Finally, sound quality sequencing reads were assembled de novo using SPAdes software version 3.9.0 (BioEasy Sdn Bhd). The Whole Genome Sequences generated were analyzed with the aid of the software EPInod (BioEasy Sdn. Bhd. Malaysia). Multi-locus Sequence Typing was conducted within the EPInod suite by streamlining all the sequences for the isolates on the MLST program against the PubMLST database (MLST version 2.6).

\section{PFGE analysis}

PFGE analysis was performed based on the standardized protocol for the subtyping of Salmonella in the PulseNet [32]. Purified DNA was digested using XbaI restriction enzyme (NEB) in a final volume of $100 \mu \mathrm{l}$ and incubated at $37^{\circ} \mathrm{C}$ for $3 \mathrm{~h}$ and embedded in a $1 \%$ SeaKem Gold Agarose (Sigma Aldrich) prepared using 0.5x TBE buffer. The reaction was run for $18 \mathrm{~h}$ using the Chef Mapper XA system (Bio-Rad) in order to resolve the DNA macro-restriction fragments. Salmonella enterica Typhimurium was used as a control. Macro-restriction patterns were compared using the FPQuest cluster analysis based on the Dice correlation coefficient, while dendograms were constructed using the unweighted-pair group method using average linkages UPGMA.

\section{Data analysis}

Salmonella isolates were assigned sequence type (ST) according to their allelic profiles corresponding to the seven housekeeping genes, while the PFGE patterns were expressed as pulsotypes. Simpson's index of diversity $(D)$, which measures the index of discrimination for the two typing methods was calculated using the formula below [18]:

$$
D=1-\frac{1}{N(N-1)} \sum_{j=1}^{S} n_{j}\left(n_{j}-1\right)
$$

According to the formula, $N$ is the total number of strains in the sample population, $S$ is the total number of types described, and $n_{j}$ is the number of strains belonging to the $j$ th type.

\section{Abbreviations}

PFGE: Pulsed Field Gel Electrophoresis; wgMLST: Whole Genome Multi-Locus Sequence Type; MDR: Multidrug-resistant; MLST: Multi-locus Sequence Typing; DNA: Deoxyribonucleic acid; $\mathrm{MOH}$ : Ministry of Health;

DVS: Department of Veterinary Services; ST: Sequence Types

\section{Acknowledgements}

The authors also wish to acknowledge the contribution of the Ministry of Health and the Department of Veterinary Services, Malaysia, for the donation of isolates and expertise during the course of this research. Finally, BioEasy Sdn Bhd for the analysis of the WGS data.

\section{Authors' contributions}

ZZ, NA, NHAH and BG analyzed WGS, PFGE data and drafted the manuscript; NS, ZS, RMA, SAH SAB and LH were responsible for sampling, isolation and characterization of the various bacterial isolates; ZZ and LH conceptualized and designed the study; all authors read and approved the final manuscript.

\section{Funding}

This work was supported by the Universiti Putra Malaysia Matching Grant (No 9300438) and Bioeasy Sdn Bhd (No 9300920). The Universiti Putra Malaysia funder consented to the publication of the manuscript but had no role in the study design, data collection and analysis. However, the BioEasy Sdn Bhd conducted WGS and analysis. BioEasy also consented on the decision to publish the manuscript.

\section{Availability of data and materials}

The datasets analyzed during the current study are available from the corresponding author on reasonable request.

Ethics approval and consent to participate

Not applicable.

\section{Consent for publication}

Not applicable.

\section{Competing interests}

The authors declare they have no competing interests.

\section{Author details}

${ }^{1}$ Institute of Bioscience, Universiti Putra Malaysia, 43400 Serdang, Selangor, Malaysia. ${ }^{2}$ Bacteriology Laboratory, Department of Veterinary Pathology and Microbiology, Faculty of Veterinary Medicine, Universiti Putra Malaysia, 43400 Serdang, Selangor, Malaysia. ${ }^{3}$ Department of Veterinary Laboratory Diagnostics, Faculty of Veterinary Medicine, Universiti Putra Malaysia, 43400 Serdang, Selangor, Malaysia. ${ }^{4}$ Food Safety and Quality Division, Ministry of Health, 62675 Putrajaya, Malaysia. ${ }^{5}$ Infectious Diseases Research Centre, Institute for Medical Research, National Institutes of Health, Setia Alam, Selangor, Malaysia. ${ }^{6}$ Diagnostic and Quality Assurance Division, Department of Veterinary Services, Ministry of Agriculture \& Agro-Based Industry, Putrajaya, Malaysia. ${ }^{7}$ Faculty of Veterinary Medicine, Usmanu Danfodiyo University, Sultan Abubakar Road, City Campus Complex, Sokoto, Sokoto State 840212, Nigeria.

Received: 14 April 2020 Accepted: 1 October 2020

Published online: 17 October 2020

\section{References}

1. Salihu MD, Magaji AA, Garba B, Saidu B, Mamuda A, Suleiman N, Wurno BS. Bacteriological quality of raw meat displayed for sale at Sokoto, Sokoto state, Nigeria. Sci J Microbiol. 2013;2:134-9.

2. Salihu M, Garba B, Isah Y. Sokoto journal of veterinary sciences evaluation of microbial contents of table eggs at retail outlets in Sokoto metropolis, Nigeria. Sokoto J Vet Sci J Vet Sci. 2015;13:22-8.

3. Abdulhaleem N, Garba B, Younis H, Mahmuda A, Hamat RA, Majid RBA, Lung LTT, Unyah NZ, Sattar A, Saidu B. Current trend on the economic and public health significance of salmonellosis in Iraq. Adv Anim Vet Sci. 2019;7: 484-91.

4. Wattiau P, Boland C, Bertrand S. Methodologies for Salmonella enterica subsp. Enterica subtyping: gold standards and alternatives. Appl Environ Microbiol. 2011;77:7877-85. 
5. Ford L, Glass K, Veitch M, Wardell R, Polkinghorne B, Dobbins T, Lal A, Kirk MD. Increasing incidence of Salmonella in Australia, 2000-2013. PLoS One. 2016;11:e0163989.

6. Heredia N, García S. Animals as sources of food-borne pathogens: a review. Anim Nutr. 2018:4:250-5.

7. Brichta-Harhay DM, Arthur TM, Bosilevac JM, Kalchayanand N, Shackelford SD, Wheeler TL, Koohmaraie M. Diversity of multidrug-resistant Salmonella enterica strains associated with cattle at harvest in the United States. Appl Environ Microbiol. 2011;77:1783-96.

8. Shah DH, Elder JR, Chiok KL, Paul NC. Genetic basis of Salmonella Enteritidis pathogenesis in chickens. In: Prod. Safe Eggs Microb. Ecol. Salmonella: Elsevier Inc:; 2017. p. 187-208.

9. Zhao S, McDermott PF, White DG, et al. Characterization of multidrug resistant Salmonella recovered from diseased animals. Vet Microbiol. 2007;123:122-32.

10. Thung TY, Radu S, Mahyudin NA, et al. Prevalence, virulence genes and antimicrobial resistance profiles of Salmonella Serovars from retail beef in Selangor, Malaysia. Front Microbiol. 2018. https:/doi.org/10.3389/fmicb.2017.02697.

11. Khaltabadi RF, Shahrokhi N, Ebrahimi-Rad M, Ehsani P. Salmonella Typhimurium in Iran: contribution of molecular and IS200 PCR methods in variants detection. PLoS One. 2019;14:e0213726.

12. Enright MC, Spratt BG. Multilocus sequence typing. Trends Microbiol. 1999;7:482-7.

13. Sharma-Kuinkel BK, Rude TH, Fowler VG. Pulse field gel electrophoresis. In: Methods Mol. Biol. Humana Press Inc.; 2016. p. 117-30.

14. Blanc DS. The use of molecular typing for epidemiologica surveillance and investigation of endemic nosocomial infections. Infect Genet Evol. 2004:4:193-7.

15. Maiden MCJ. Multilocus sequence typing of Bacteria. Annu Rev Microbiol. 2006:60:561-88.

16. Wang J, Li J, Liu F, Cheng Y, Su J. Characterization of Salmonella enterica isolates from diseased poultry in northern China between 2014 and 2018. Pathogens. 2020;9:95.

17. Larsen MV, Cosentino S, Rasmussen S, et al. Multilocus sequence typing of total-genome-sequenced bacteria. J Clin Microbiol. 2012;50:1355-61.

18. Hunter PR, Gaston MA. Numerical index of the discriminatory ability of typing systems: an application of Simpson's index of diversity. J Clin Microbiol. 1988;26:2465-6.

19. Nemoy LL, Kotetishvili M, Tigno J, et al. Multilocus sequence typing versus pulsed-field gel electrophoresis for characterization of extended-spectrum beta-lactamase-producing Escherichia coli isolates. J Clin Microbiol. 2005;43: $1776-81$.

20. Brunelle BW, Bearson SMD, Bearson BL. Tetracycline accelerates the temporallyregulated invasion response in specific isolates of multidrug-resistant Salmonella enterica serovar Typhimurium. BMC Microbiol. 2013;13:202.

21. Azmi M, Hassali A, Rhu H, Ashutosh Y, Verma K, Hussain R, Sivaraman S. Antibiotic use in food animals: Malaysia overview. School of Pharmaceutical Sciences Universiti Sains Malaysia 11800 Minden, Penang, Malaysia., Penang, Malaysia; 2018.

22. Manyi-Loh C, Mamphweli S, Meyer E, Okoh A. Antibiotic use in agriculture and its consequential resistance in environmental sources: potential public health implications. Molecules. 2018. https://doi.org/10.3390/ molecules23040795

23. Alikhan N-F, Zhou Z, Sergeant MJ, Achtman M. A genomic overview of the population structure of Salmonella. PLoS Genet. 2018;14:e1007261.

24. Aung $C$, Chau, et al. Salmonella in retail food and wild birds in Singapore-prevalence, antimicrobial resistance, and sequence types. Int J Environ Res Public Health. 2019;16:4235.

25. Adesiji YO, Deekshit VK, Karunasagar I. Antimicrobial-resistant genes associated with Salmonella spp. isolated from human, poultry, and seafood sources. Food Sci Nutr. 2014;2:436-42.

26. Castellanos LR, Van Der Graaf-Van Bloois L, Donado-Godoy P, et al. Genomic characterization of extended-spectrum cephalosporin-resistant Salmonella enterica in the Colombian poultry chain. Front Microbiol. 2018. https://doi. org/10.3389/fmicb.2018.02431.

27. Bing LW, Liu B, Na ZX, Jing YS, Ming SX. Diversity of Salmonella isolates using serotyping and multilocus sequence typing. Food Microbiol. 2011 28:1182-9.

28. Wiesner M, Zaidi MB, Calva E, Fernández-Mora M, Calva JJ, Silva C Association of virulence plasmid and antibiotic resistance determinants with chromosomal multilocus genotypes in Mexican Salmonella enterica serovar Typhimurium strains. BMC Microbiol. 2009;9:131.

29. Stepan RM, Sherwood JS, Petermann SR, Logue CM. Molecular and comparative analysis of Salmonella enterica Senftenberg from humans and animals using PFGE, MLST and NARMS. BMC Microbiol. 2011. https://doi.org/ 10.1186/1471-2180-11-153.

30. Shaaly A, Tellevik MG, Langeland N, Høiby EA, Jureen R. Comparison of serotyping, pulsed field gel electrophoresis and amplified fragment length polymorphism for typing of Streptococcus pneumoniae. J Med Microbiol. 2005;54:467-72.

31. Lubbers B V, Papich MG, Schwarz S, et al (2018) Performance standards for antimicrobial disk and dilution susceptibility tests for Bacteria isolated from animals a CLSI supplement for global application. Performance Standards for Antimicrobial Disk and Dilution Susceptibility Tests for Bacteria Isolated From Animals.

32. Ribot EM, Fair MA, Gautom R, Cameron DN, Hunter SB, Swaminathan B, Barrett TJ. Standardization of pulsed-field gel electrophoresis protocols for the subtyping of Escherichia coli 0157:H7, Salmonella, and Shigella for PulseNet. Foodborne Pathog Dis. 2006:3:59-67.

\section{Publisher's Note}

Springer Nature remains neutral with regard to jurisdictional claims in published maps and institutional affiliations.

Ready to submit your research? Choose BMC and benefit from:

- fast, convenient online submission

- thorough peer review by experienced researchers in your field

- rapid publication on acceptance

- support for research data, including large and complex data types

- gold Open Access which fosters wider collaboration and increased citations

- maximum visibility for your research: over $100 \mathrm{M}$ website views per year

At $\mathrm{BMC}$, research is always in progress.

Learn more biomedcentral.com/submissions 